\title{
Subject Index Vol. 17, 1994
}

ACOMED regimen 172 Acute myelogenous leukemia, children 166 myeloid leukemia 158,162

Adjuvant chemotherapy 2 134,3 242

Afterloading 6604

All-trans retinoic acid (ATRA) 5515 Anal canal carcinoma 4398

- $\quad$ margin carcinoma 4398

Angiogenesis 135,144

Angiosarcomas 3242

Antiestrogens Suppl. 127, Suppl. 132 ,

Suppl. 1 40, Suppl. 1 45, Suppl. 149 Aspiration 4420 Auto-antibodies 4402

Benign prostatic hyperplasia 5478 Biological response modifiers 3216 Biopsy, needle 4420

Bladder cancer 5523 Blood circulation 2174 Bone marrow micrometastases 2184 Brain tumour marker 6600 BRCA-1 5470

Breast cancer 116,121,4402,4410, Suppl. 145

- cells Suppl. 122

-, genetic predisposition 5470

-, node-negative, grading 2166

-, prognostic factors 144

neoplasms 3242 Breast-conserving therapy 4410 Bryostatin 13234

Cancer 4358

prevention 5470

prognosis 4358

therapy 4358 Carcinoma in situ 2150

-, prostate, early detection 5478

-, uterine cervix 6604

c-erbB-2 4346

c-erbB-3 4346

c-erbB-4 4346

Cell adhesion 5528

Cerebrospinal fluid 2180

Cervical intraepithelial neoplasia 2150

c-fos Suppl. 127

Chemosensitizers 18

Chemotherapeutic agents 3234

Chemotherapy $150,158,162,178$

-, taxol 186

CHOEP regimen 169

Cisplatin(um) 2 154,5 498

Clinical research units 6630 
c-myc Suppl. 127

Colon adenocarcinoma 5528

Color Doppler sonography 135

Colorectal cancer 2 184,4 368,4 434

carcinoma 178 Combined modality therapy 5498

radiochemotherapy 4 398, 6612 Conization 2150 Consolidation 162 Corticosteroid therapy 2

158 Cranial irradiation 166 Cutaneous reactions 2158 Cytodiagnosis 4420

Cytosine arabinoside 2158

Desmoid tumours 4376 Dexverapamil 6572 Doctor-patient relationship 6624 Dose

intensification 169,3216

- $\quad$ intensity 3248

Droloxifene Suppl. 1 6, Suppl. 117,

Suppl. 1 22, Suppl. 1 32, Suppl. 1 40, Suppl. 1 45, Suppl. 149 Drug intensity 5486

- $\quad$ therapy 3216

Early and late radiation effects 6612 EGF receptor 4346 Elderly tumor patient 4410 Epidermal growth factor receptor prognosis

2166 Esophageal carcinoma 5498 Etoposide 2154 Extracellular matrix 5528

Flexible applicator 6604 Fludarabine 5508 5-Fluorouracil 2 154,4 368, 5498 Folinicacid 178,2

154 Follow-up 2150

Galactosyltransferases 2180 Gastric cancer 2154 Gastrointestinal lymphomas 4384

toxicity 3278 G-CSF 169,3248 Gene therapy, aims 191

-, limits 191 Genetic counseling 5470

differences 5523

markers 5470 Genotype 3212

German MTC Study Group 6594 Glutathione S-transferase 128 Glycoprotein 170128 P-

Glycoprotein 18 GM-CSF priming 158 Goal of treatment 4454 Good clinical practice 6630

Growth factors Suppl. 127

- $\quad$ inhibition Suppl. 117

Haematopoietic growth factors 3216 Hepatocellular tumor Suppl. 16 High-grade malignant non-Hodgkin

lymphomas 1 69,1 72 Histomorphology 3212 HLA typing 5523

IGF-binding proteins 6600 Illness paradigm 6624 Ilmofosine 3278 Immunocytic lymphoma 5

508 Immunocytochemistry 2184 Immunophenotype 3212 Immunosuppression 5508

Implementation in Germany 6630 Induction chemotherapy 3294 Insulin-like growth factors 6

600 Intensive chemotherapy 172 Interferon 178,5 486 Interferon- $\alpha 3$ 254,3 263 Interleukin-2 3

254,3 263 Interleukin-6 3263 Intermittent therapy Suppl. 122 Intracavitary radiotherapy 6604

Laparoscopic colectomy 3226 Laparoscopy 3226 Ligands 4346 Lobaplatin 2142 Local

treatment 116 Long-term results 4439 Lung cancer 128

-, diagnostic procedure 2124

-, prognostic factors 2124

-, staging 2124

metastases 4439 Lymphadenectomy 2134 Lymphocyte activation 2114

Malignancy 4391

Malignant pericardial effusion 5504

MALT lymphomas 4384

Mastectomy 4410 
MCF-7 Suppl. 117

- $\quad$ cells Suppl. 127

Mediastinal large B-cell lymphoma 3212 Medullary thyroid carcinoma 6594 Metastasis 2114

Metastatic breast cancer 186 , Suppl. 132 ,

Suppl. 140 Microvessel density 144

quantitation 135 Miltefosine 116

solution 121

Mitoxantrone, intrapericardial instillation

5504 Modulation 4368 -, MDR 6572

649

Multidrug resistance 6572

- $\quad$-, reversal 18

Multi-modal treatment concept 3294 Multiple drug resistance 128

- $\quad$ myeloma 4428

Myelodysplastic syndrome 5515

Neovascularisation 144

Non-Hodgkin lymphoma(s) 3 248,4 428,

5486 Non-small cell lung cancer 3294 Non-steroidal anti-inflammatory drugs

4376

Oestrogen receptors 4376 Ovarian cancer 2134

- $\quad-$, life quality 150

p53 4402 Patients at risk 172 Pericardiocentesis 5504 Peripheral blood stem cells 3216

Pharmakokinetics Suppl. 149 Phase I study 2142

II study 186,2 154,3 278 Postmenopausal status 2174 Premenopausal status 2174 Pretreated patients Suppl. 140 Primary therapy Suppl. 145 Prognostic factors 121,4 439, 6594

marker 4428 Prolactinoma 6600 Prostate cancer 6578 Prostate-specific antigen 6578 Prostatic carcinoma 6586

Proteases 4358

Protein biosynthesis Suppl. 117

- $\quad$ kinase modulators 3234

PSA 5478

Pulmonary edema, toxic 2158

- $\quad$ toxicity 2158

Quality assurance 4454

- $\quad$ of life $178,4410,5547$

Quantification methods 5528

Radiation 5498

- $\quad$ therapy 4398

Radiotherapy 6 578,6 586

Rat Suppl. 16

Rate of infections 5508 Rehabilitation 5547 Remission induction methods 5486 Renal cell cancer 3263,3278

- carcinoma 3254

failure 4428 Retinoids 5515 Risk factors 5486 
Salutogenesis 6624 Sarcoma(s) 3 242,4 391 -, diagnosis 4391 -, treatment 4391 Second-look laparotomy 2134 Serum B2-microglobulin 4428 Signal transduction 3234 Skin metastases 121 Soft-tissue sarcoma 3216 Solid tumors 6612 S-phase fraction 2166

Stage D1 prostatic cancer 6586

Staging laparoscopy 3226

Stereology 135

Stomach 4391

Study AML·BFM-87 166

Surgery of metastases 4439

Surgical therapy 6594

Tamoxifen 4 376, Suppl. 1 6, Suppl. 149

Taxol 186

Therapy 4384

Thrombocytopenia 2142

Tissue polypeptide-specific antigen 3270

Topical chemotherapy 116

- $\quad$ treatment 121

Toxicity 6612

TPA 3270

Treatment 2150

-, mediastinal large B-cell lymphoma

3212 Tumor biology 4358

marker 2 180,3 270,5 478

stage III, NSCLC 3294 Tumours of uterus and ovary 2174

Ultrasonography 4420 Unconventional cancer therapy 6624 Urinary neopterin 4434 Uterine cervix 2150

Variants of CD44 2114

Waldenström's macroglobulinemia 5508 Well-being questionnaire 150

650

Subject Index Vol. 17,1994 